\title{
The impact of dose of the St. Louis Healthy Start program and prenatal care adequacy on birth outcomes
}

\author{
Beth Rotter ${ }^{* 1,2}$, Michael Elliott ${ }^{2}$, Angela Recktenwald ${ }^{3}$, Darcy Scharff ${ }^{2}$ \\ ${ }^{1}$ BJC Healthcare, St. Louis, MO, United States \\ ${ }^{2}$ College for Public Health and Social Justice, Saint Louis University, St. Louis, MO, United States \\ ${ }^{3}$ Nurses for Newborns Foundation, St. Louis, MO, United States
}

Received: September 10, 2015

Accepted: October 18, 2015

Online Published: November 11, 2015

DOI: $10.5430 /$ jnep.v6n2p123

URL: http://dx.doi.org/10.5430/jnep.v6n2p123

\begin{abstract}
The federal Healthy Start program began over 20 years ago to reduce disparities in infant mortality. Data on outcomes of the program, in particular related to the amount of exposure to Healthy Start, are lacking in the literature. This study was designed to assess the impact of dose of a Healthy Start program on prenatal care adequacy (defined using the Kotelchuck Index) and birth outcomes. Two hundred and thirty African American women were included in the study sample. Participants were women served by their local Healthy Start program between 2007 and 2012. Traditional multivariable logistic and linear regression analyses were employed to determine the impact of the Healthy Start program. Dose of Healthy Start (OR $=1.296,95 \%$ CI $[1.060,1.585])$ was found to be positively predictive of prenatal care adequacy. Because significant interaction effects were found between dose and prenatal care adequacy, the sample was then stratified by level of prenatal care adequacy, and dose was assessed as an independent variable on birth outcomes (infant birth weight and pre-term birth). Dose was negatively predictive of infant birth weight $(\beta=-60.015, p<.10)$ among women with adequate plus prenatal care; dose was positively predictive of pre-term birth $(\mathrm{OR}=1.427,90 \% \mathrm{CI}[1.104,1.845])$ among women with adequate plus prenatal care. Dose was found to be an important predictor of birth outcomes across varying categories of prenatal care adequacy and probably works synergistically through prenatal care. Future research should continue to explore these relationships at each level of prenatal care adequacy.
\end{abstract}

Key Words: Healthy start, Birth outcomes, Infant mortality, Low birth weight, Pre-term birth, Prenatal care adequacy, Logistic regression, Linear regression

\section{INTRODUCTION}

Infant mortality continues to be a major public health concern. The rates of infant mortality remain high, particularly in African American communities. ${ }^{[1-6]}$ African American infants are more than twice as likely to die during their first year of life when compared to White infants. ${ }^{[2-4]}$
Predictors of infant mortality include low birth weight, preterm birth and inadequate prenatal care. ${ }^{[5]}$ Rates of these predictors are also higher in African American women when compared to White women. ${ }^{[6]}$ African American women in the United States are more than twice as likely to deliver a low birth weight baby $(13.16 \%$ vs. $6.96 \%),{ }^{[7,8]}$ and experience higher proportions of pre-term births (13.23\%)

*Correspondence: Beth Rotter; Email: brotter@bjc.org; Address: College for Public Health and Social Justice, Saint Louis University, St. Louis, MO, United States.

Published by Sciedu Press 
compared to White women $(8.9 \%) .{ }^{[8]}$ In addition, $10.0 \%$ of African American women receive inadequate prenatal care, compared to $4.4 \%$ of White women. ${ }^{[9]}$ These differences are even more pronounced in urban areas that have concentrated proportions of African Americans and other underrepresented minorities. ${ }^{[6,10]}$

For over 20 years, various maternal/child health initiatives have been implemented to improve birth outcomes in African American communities. One in particular, Healthy Start (HS), began in 1991 to target 15 communities where infant mortality rates were of most concern. ${ }^{[10]}$ Currently, there are $105 \mathrm{HS}$ programs in 39 states. The program operates through monthly home visits by nurses and community health worker's (CHW's), comprehensive prenatal and postpartum health education, referrals and ongoing assistance to reduce disparities and ensure healthy birth outcomes. ${ }^{[2,10]}$ Home visiting is an important component of HS programs; this mixed-model approach utilizes both CHW's and nurses to provide coordinated in-home case management for women and their families.

Previous evaluation studies of HS programs have primarily focused on process and short-term impacts. Few studies have published the impact of HS on birth outcomes, and of these, various methodological approaches to creating comparison groups have been used. ${ }^{[1,11-15]}$ One study employed propensity score matching using a retrospective case-control design to assess the impact of the HS program on birth outcomes. This study found significant beneficial group differences for low birth weight and pre-term birth; however, outcomes were only assessed among clients who received inadequate prenatal care. ${ }^{[15]}$ More recently in 2014, another study employed propensity score matching, and found that African American HS participants delivered higher birth weight infants than non HS participants. Despite matching on several demographic characteristics, this study only included women who initiated prenatal care within their first trimester. ${ }^{[1]}$ While both studies are promising, more work is needed to assess the impact of HS programs on birth outcomes at all levels of prenatal care adequacy (inadequate, intermediate, adequate and adequate plus)

Previous evaluations focused on the overall impact of HS and have not explored how specific HS program components are responsible for impacting birth outcomes. In particular, no HS programs have been evaluated for the impact of dose of in-home case management on birth outcomes across all levels of prenatal care adequacy. To date, only one study (a non-Healthy Start intervention) assessed the impact of dose of a prenatal home visiting program on pregnancy outcomes. In this study, Slaughter and Issel defined dose as a combi- nation of duration of enrollment, amount of time spent with a case manager, and breadth of interventions. The findings indicated that a higher dose of in-home prenatal case management was significantly associated with reductions in low birth weight babies and pre-term births. While Slaughter and Issel controlled for prenatal care adequacy using the Kotelchuck scale, the researchers did not stratify by level of prenatal care adequacy to measure direct impact of level of prenatal care on birth outcomes. ${ }^{[16,17]}$

This study assessed the relationship between dose of in-home case management and prenatal care adequacy as defined by prenatal (medical) visits. The purpose of this study was to determine the impact of dose of HS in-home case management on prenatal care adequacy. Additionally, this research further explored the impact of dose of in-home case management on birth outcomes, independent of prenatal care adequacy. This study had three research questions: (1) How does dose of HS in-home case management relate to prenatal care adequacy? (2) How does level of prenatal care adequacy moderate the impact of dose of HS in-home case management on infant birth weight? and (3) How does level of prenatal care adequacy moderate the impact of dose of HS in-home case management on pre-term birth?

\section{MeTHODS}

\subsection{Study population}

The data used for this research were secondary data collected from a longitudinal database managed by a local non-for profit agency. Data were obtained from women enrolled in the Saint Louis Healthy Start (SLHS) program between 6/1/2007 and 5/31/2012. All cases were Medicaid eligible, African American pregnant women who were at risk for poor birth outcomes due to medical and/or social risk factors. In addition, they lived in one of three zip codes (63113, 63120, 63136). The initial sample size consisted of 451 cases. Exclusion criteria were cases with less than two Healthy Start visits $(n=57)$; these cases would have had no corresponding birth outcomes because the first visit was an intake assessment. Additionally, other cases with no associated birth data were excluded, as these cases were those that self-terminated from the program $(n=33)$. Cases that had an older child who had already graduated or terminated from the SLHS program were also excluded $(n=43)$. In cases with multiple children, any subsequent children who received SLHS services were removed in an effort to measure true program exposure $(n=48)$. Finally, cases that were referred in the third trimester and/or received less than two prenatal care visits were excluded ( $n=40)$; this was done in an effort to measure true program effects. Two hundred and thirty (230) women were included in the final analysis. The Institutional 
Review Board at Saint Louis University approved this study.

\subsection{Measures}

The variables used in this study included dose of SLHS in-home case management, duration in program, prenatal care adequacy, birth outcomes (infant birth weight, pre-term birth), and maternal characteristics (age, education).

Dose of SLHS in-home case management was defined as the average of prenatal nurse and $\mathrm{CHW}$ home visits (that provide information and referral support) that each client experienced while enrolled. The average was calculated by adding the number of nurse and CHW visits and dividing by two. SLHS nurses are required to visit clients at least monthly, although if women are identified as high risk, their visit frequency may increase to bi-monthly; community health workers supplement the nurse visits and are provided in the home and over the telephone. Duration was calculated by subtracting the date the client was referred into the SLHS program from the baby birth date (measured in days). Prenatal care adequacy was defined using the Kotelchuck Index. This index includes four categories (inadequate, intermediate, adequate, adequate plus), and combines the timing of the first prenatal (medical) visit with the ratio of observed to expected number of prenatal care visits based on prenatal care standards set forth by the American Congress of Obstetrics and Gynecologists (ACOG). ${ }^{[17]}$ Birth weight was measured in grams and low birth weight was defined as any infant born weighing less than 2,500 grams (5 lbs. 8 oz.). Pre-term birth was defined as any birth occurring prior to 37 weeks of gestation (yes/no). Age was measured as a continuous variable, in years. Maternal education was categorized as: $0=$ did not finish high school, referent category; 1 = high school; 2 = beyond high school.

\subsection{Statistical analysis}

Descriptive statistics were calculated for categorical and continuous variables, multivariable logistic and linear regression techniques were conducted to assess program impact. Factor analysis was conducted to determine whether variables included in dose (average number of nurse and community health worker home-visits) were related enough to be validly combined. Statistical analysis was conducted using SPSS version 18.0. ${ }^{[18,19]}$ Maternal characteristics (age, education) were non-significant in all models, and were therefore removed from all analyses.

The first research question was two-tailed, and alpha was set at 0.05 . Prenatal care adequacy was converted to a binary outcome variable, with adequate plus and adequate levels of prenatal care $=$ yes, and intermediate and inadequate prenatal care $=$ no. For the second and third research questions, two-sided tests were employed, and the significance level was set a priori at alpha equal to 0.10 due to the limited sample size and exploratory nature of this study. Data were stratified by each of the four levels of prenatal care adequacy for research questions two and three since dose and prenatal care adequacy had significant interaction effects in both of these regression models. Logistic regression was used for research questions one and three, while linear regression was employed for research question two. Power analysis found that for all research questions, the sample size of 230 participants was more than adequate for the number of predictors included in the regression models. ${ }^{[20-22]}$ In addition, the SPSS bootstrapping method was applied to provide robust estimates of effect due to small sample size. ${ }^{[18]}$

\section{Results}

\subsection{Sample characteristics}

This study sample included 230 women who had participated in SLHS during 2007-2012. The mean age of women in the sample was 20.99 (range of 13-37, SD = 5.452). Approximately half $(46.5 \%)$ did not finish high school, while a quarter $(25.2 \%)$ completed high school. Only about another quarter $(27.9 \%)$ had education levels beyond high school. Education data was missing from one participant $(0.4 \%)$. All participants were African American. On average, women received 3.79 prenatal SLHS visits (range 2-11, SD $=2.38$ ). Across the four levels of prenatal care adequacy, $12.6 \%$ women $(n=29)$ had inadequate prenatal care, $12.6 \%$ women $(n=29)$ intermediate prenatal care, $33.9 \%$ women $(n=78)$ adequate prenatal care, and $40.9 \%$ women $(n=94)$ had adequate plus prenatal care (see Table 1).

\subsection{Outcomes}

Factor analysis for the dose variable resulted in a Cronbach's alpha of 0.676 , which is approaching the acceptable value of 0.7. ${ }^{[18]}$ Therefore, we determined that the dosage variable used in this study displayed an acceptable level of internal consistency.

Dose of SLHS in-home case management was found to be a significant positive predictor of prenatal care adequacy (OR $=1.296,95 \%$ CI $[1.060,1.514]), p<.05$. Next, we wanted to determine if dose of SLHS in-home case management impacted birth outcomes (birth weight, pre-term birth). Because significant interaction effects were found between dose of SLHS in-home case management and prenatal care adequacy $(p=.033)$, we stratified by prenatal care adequacy, and removed the interaction term. In order to assess the direct impact of dose of SLHS in-home case management on birth weight, duration in program was controlled for to ensure true dosage effects were being measured across participants with 
varying time in the SLHS program. For women with inadequate, intermediate and adequate prenatal care, dose was not a significant predictor of infant birth weight. However, for women who obtained adequate plus prenatal care, dose of SLHS in-home case management was a significant predictor of infant birth weight; $\beta$ (SE) $=-60.015$ (33.667), $p=.071$ (see Table 2).

Again, stratifying by level of prenatal care adequacy and controlling for duration, we found that among women with inadequate, intermediate and adequate plus levels of prenatal care, dose of SLHS in-home case management was not a significant predictor of preterm birth. However, for women who obtained adequate plus prenatal care, dose (OR $=1.427,90 \%$ CI $[1.104,1.845])$ was a significant predictor for pre-term birth (see Table 3).
Table 1. Demographic characteristics of healthy start participants (2007-2012)

\begin{tabular}{llll}
\hline & N & \% & Mean (SD) \\
\hline Age & 230 & & $20.99(5.452)$ \\
Education & & & \\
$\quad$ Did not finish high school & 107 & 46.5 & \\
$\quad$ High school & 58 & 25.2 & \\
$\quad$ Beyond high school & 64 & 27.9 & \\
$\quad$ Missing & 1 & 0.04 & \\
Race & & & \\
$\quad$ African American & 230 & 100.0 & \\
Prenatal Care Adequacy & & & \\
$\quad$ Inadequate & 29 & 12.6 & \\
$\quad$ Intermediate & 29 & 12.6 & \\
$\quad$ Adequate & 78 & 33.9 & \\
$\quad$ Adequate Plus & 94 & 40.9 & \\
\hline
\end{tabular}

Table 2. Relationship of dose and duration and low birth weight (grams) stratified by prenatal care adequacy

\begin{tabular}{lllll}
\hline Variable & $\begin{array}{l}\text { Inadequate }(\mathbf{n}=\mathbf{2 9}) \\
\boldsymbol{\beta}\end{array}$ & $\begin{array}{l}\text { Intermediate }(\mathbf{n}=\mathbf{2 9}) \\
\boldsymbol{\beta}\end{array}$ & $\begin{array}{l}\text { Adequate }(\mathbf{n}=\mathbf{7 8}) \\
\boldsymbol{\beta}\end{array}$ & $\begin{array}{l}\text { Adequate Plus }(\mathbf{n}=\mathbf{9 4}) \\
\boldsymbol{\beta}\end{array}$ \\
\hline Intercept & $3,739.955$ & 13.194 & $2,642.503$ & $2,146.827$ \\
Dose & 85.080 & 0.977 & -2.876 & $-60.015^{*}$ \\
F & 1.079 & -0.003 & 2.705 & 2.898 \\
$R^{2}$ & 0.011 & & 0.081 & 0.075 \\
\hline$* p<.10$ & & &
\end{tabular}

Table 3. Relationship of dose and duration and pre-term birth (yes/no) stratified by prenatal care adequacy

\begin{tabular}{|c|c|c|c|c|}
\hline Variable & $\begin{array}{l}\text { Inadequate }(\mathrm{n}=29) \\
\text { OR }(90 \% \mathrm{CI})\end{array}$ & $\begin{array}{l}\text { Intermediate }(n=29) \\
\text { OR }(90 \% \text { CI })\end{array}$ & $\begin{array}{l}\text { Adequate }(\mathrm{n}=77,1 \text { missing) } \\
\text { OR }(90 \% \text { CI })\end{array}$ & $\begin{array}{l}\text { Adequate Plus ( }=90,4 \text { missing) } \\
\text { OR }(90 \% \text { CI) }\end{array}$ \\
\hline Intercept & -3.474 & 2.648 & 0.32091 & -2.538 \\
\hline Dose & $0.593(0.261,1.346)$ & $0.568(0.223,1.448)$ & $1.041(0.426,2.543)$ & $1.427(1.104,1.845)^{*}$ \\
\hline$\chi^{2}$ & $1.496(3)$ & $3.002(3)$ & $8.538(4)^{*}$ & $11.677(3)^{*}$ \\
\hline
\end{tabular}

\section{DISCUSSION AND CONCLUSION}

This study evaluated the impact of dose of SLHS in-home case management on prenatal care adequacy and birth outcomes (birth weight, preterm birth; stratified by levels of prenatal care adequacy). First, we determined the relationship between dose of SLHS in-home case management and prenatal care adequacy. As dose increased, the odds of obtaining adequate prenatal care also increased. This finding suggests that nurses who are completing frequent prenatal in-home case management visits may be consistently encouraging mothers to obtain prenatal care visits with their medical providers, potentially increasing their odds of obtaining adequate prenatal care.

In this research, there was great variability in the number of in-home case management visits for clients who obtained adequate prenatal care (majority of women received between 7-11 visits) when compared to cases who obtained inadequate prenatal care (majority of women received between 2-6 visits). This variability suggests the need to explore how much SLHS case management (tailored dose) is required to meet the individual needs and situation of the SLHS clients. Slaughter and Issel (2012) report that, to date, the "gold standard" of prenatal in-home case management has not yet been established; however, they believe that a minimum threshold does exist and more research is warranted to identify that threshold. ${ }^{[16]}$ Future research should assess measures of dose to uncover a possible "tipping" point that is associated with obtaining adequate prenatal care. In addition, future measures of dose might consider including a measure of receptivity of SLHS clients to understand concerns around 
uptake and retention into the program, both of which have an impact on dose. We suggest that the amount and type of SLHS dose may need to be tailored to individual clients and evaluation research that tailors SLHS dose to individuals is needed. Findings would result in significant implications on program planning, reach, resource allocation, as well as implications for funding and policy.

Next, we explored the impact of dose of SLHS in-home case management on infant birth weight and preterm birth, stratifying by the four levels of prenatal care adequacy. For cases with adequate plus prenatal care, dose was negatively related to infant birth weight, indicating that as dose increases, infant birth weight decreased. This finding is counterintuitive and opposite of what has been found in the literature related to dose of case management. Slaugther and Issel found that women who received high doses of prenatal case management were $40 \%$ less likely to have a low birth weight baby. ${ }^{[16]}$ The opposite finding from the current study suggests that there may be additional unmeasured variables that are causing a mother to identify as high-risk, for example social factors such as poverty, unemployment, or unstable housing, which were unaccounted for in this study. Once identified as high-risk status, the mother is automatically eligible to receive additional prenatal care that would place her in the adequate plus prenatal care category. These elevated risk mothers would also be queued to receive additional SLHS case management visits. Social issues need to be accounted for in future analyses to determine the impact they might have on prenatal care and birth outcomes.

As the dose of SLHS in-home case management increased among women who obtained adequate plus prenatal care, the odds of having a pre-term birth increased. This finding is also opposite of what has been found in the literature. Research has shown that as prenatal care increases, the risk for pre-term birth decreases. ${ }^{[16]}$ Again, it is probable that unmeasured variables (e.g., social or behavioral factors such as poverty, prenatal nutrition, and tobacco) could be accounting for increased rates of pre-term births. It is also likely that when these cases were identified early-on as high-risk pregnancies, they automatically received an increased number of SLHS case management and prenatal care visits because of this status. It should be noted that although sample sizes in the four prenatal care categories were small following stratification, there was adequate power to detect differences. Further analysis should continue to use adequate sample sizes and statistically control for additional social and/or behavioral factors across all levels of prenatal care adequacy.

This research was the first study of its kind to study dose of in-home case management in a SLHS program across the four levels of prenatal care adequacy and its impact on birth outcomes. Huber and colleagues indicate the importance of establishing a mechanism for assessing dose, and argue that doing so can have impacts on guiding practice, advancing the research agenda, and allowing for healthcare providers to determine the effectiveness of their interventions. ${ }^{[23]}$ Findings from this study support previous literature that in-home case management results in increased prenatal care adequacy. ${ }^{[24,25]}$ This research began to uncover why prenatal case management is an effective intervention by establishing a positive relationship between dose of SLHS in-home case management and obtaining adequate prenatal care. This finding is exciting, and future research should continue to explore thresholds of dose in relation to obtaining adequate prenatal care. If such a relationship is established, future SLHS programs can tailor dose of case management for high risk clients in the hopes of improving prenatal care and birth outcomes.

This research also found that dose of SLHS in-home case management functions differently across varying levels of prenatal care on birth outcomes. These findings raise new questions about where case management resources should be focused in order to obtain maximum benefits, and how much SLHS case management is enough to yield positive birth outcomes. While our findings were counter to existing literature, we recognize that our sample sizes in the stratified sub-analyses were very small, and possibly less likely to detect appropriate significant differences. We were also unable to control for factors related to additional in-home case management visits and high-risk pregnancies. Future studies should control for additional factors that queue women to receive additional in-home case management visits.

Healthy Start programs should continue to evaluate outcomes and program fidelity to better understand the relative impact of the components of SLHS on birth outcomes. Recognizing that SLHS is a community-based program, and will have unique aspects related to the community being served, it is very important to measure fidelity of SLHS program components as a way to account for variations in context and content of SLHS programs. For example, the SLHS program evaluated in this research employed CHW's, in collaboration with nurses, for in-home case management. The use of CHWs is not a SLHS program requirement, and differs from program to program. In this research, a mixed-model approach defined our measure of dose of case management, so it may be important for future studies to look carefully at fidelity of dose of case management to understand the impact, and potentially added benefits, that using $\mathrm{CHW}$ 's can have on obtaining adequate prenatal care and/or improving birth outcomes. 
Future researchers may also want to determine receptivity (e.g., clients openness to participate, motivation), as well as level of engagement in the SLHS program. This research did not explore receptivity to nor engagement in the SLHS program, both of which may be associated with dose of in-home case management. One study in 2007 did explore engagement of a SLHS program, and found that women offered various reasons for participating in the program, including job training, social support, prenatal nutritional information, post-partum baby care, and transportation to physician appointments. ${ }^{[26]}$ Future research should explore engagement, barriers to engagement, as well as client receptivity to SLHS case management so that the appropriate amount and type of dose can be applied in a variety of contexts and communities.

The results from this research are promising. This study found that an increased dose of case management resulted in obtaining adequate prenatal care. We believe that case management alone does not have an impact on birth outcomes, but works synergistically through prenatal care. Therefore, nurses providing in-home case management should assure that they advise clients to obtain early and adequate prenatal care. This finding is important because it supports the need to continually fund SLHS programs in high-risk communities to assure that women get the required and appropriate prenatal care and benefit from positive birth outcomes.

\section{ACKNOWLEDGEMENTS}

This research was supported by a partnership between the St. Louis Maternal, Child and Family Health Coalition, Nurses for Newborns and Saint Louis University. The authors would like to thank Lora Gulley, Kendra Copanas, Ron Tompkins, and Melinda Ohlemiller for their support of this research. This collaboration and continued work is funded through a Health Resources and Services Administration Grant (SLU eRS\# 13712).

\section{Conflicts of Interest Disclosure}

The authors declare that there is no conflict of interest.

\section{REFERENCES}

[1] Kothari CL, Zielinkski R, James A, et al. Improved birth weight for Black infants: Outcomes of a Healthy Start Program. American Journal of Public Health. 2014; 104(Suppl 1): S96-S104. PMid:24354844 http://dx.doi.org/10.2105/AJPH. 2013.301359

[2] National Healthy Start Association. Federal healthy start initiative: A national network for effective home visitation and family support services. 2010

[3] MacDorman MF, Hoyert DL, Mathews TJ. Recent declines in infant mortality in the United States, 2005-2011. NCHS data brief, no 120 Hyattsville, MD: National Center for Health Statistics. 2013.

[4] Maternal, Child and Family Health Coalition. St. Louis fetal infant mortality review program annual report. 2006. Available from: http://www.stl-mcfhc.org/images/docs/FIMR_Annu al_Report_2006.pdf

[5] Beck LF, Morrow B, Lipscomb LE, et al. Pregnancy risk assessment monitoring system (PRAMS), 1999. Morbidity and Mortality Weekly Report. 2002; 51(SS02): 1-26.

[6] Flick LH, Leet TL. Monitoring Infant Mortality in St. Louis City and County: Update for 2000-2004. Final Report submitted to the St Louis Maternal, Child and Family Health Coalition. 2007.

[7] The Centers for Disease Control and Prevention, National Vital Statistics Reports. Births: Final Data for 2010. Available from: http: //www.cdc.gov/nchs/data/nvsr/nvsr61/nvsr61_01.pdf

[8] Hamilton BE, Martin JA, Ventura SJ. Births: Preliminary Data for 2014. National vital statistics reports. Hyattsville, MD: National Center for Health Statistics. 2015.

[9] Percentage of Mothers Receiving Late or No Prenatal Care, by Race and Hispanic Origin. 2013. Available from: http://www . childt rends.org/wp-content/uploads/2014/07/25_fig2.jpg

[10] National Healthy Start Association. Available from: http://www. nationalhealthystart.org/healthy_start_initiative
[11] Salihu HM, Mbah AK, Jeffers D, et al. Healthy Start Program and feto-infant morbidity outcomes: Evaluation of program effectiveness. Maternal \& Child Health Journal. 2009; 13(1): 56-65. PMid:18690524 http://dx.doi.org/10.1007/s10995-008-0 400-y

[12] Brand A, Walker D, et al. Intermediate Outcomes, Strategies, and Challenges of Eight Healthy Start Projects. Maternal and Child Health Journal. 2010; 14(5): 654-665. PMid:19011959 http:// dx.doi.org/10.1007/s10995-008-0421-6

[13] Rosenbach M, O'Neil S, Cook B, et al. Characteristics, Access, Utilization, Satisfaction, and Outcomes of Healthy Start Participants in Eight Sites. Maternal \& Child Health Journal. 2010; 14(5): 666-679. PMid:19590941 http://dx.doi.org/10.1007/s10995-009-0 474-1

[14] Meghea CI, Raffo JE, VanderMeulen P, et al. Moving Toward Evidence-Based Federal Healthy Start Program Evaluations: Accounting for Bias in Birth Outcomes Studies. American Journal of Public Health. 2014; 104(S1): S25-7. PMid:24354826 http: //dx.doi.org/10.2105/AJPH. 2013.301276

[15] Cooper B, Scharff D, Elliot M, et al. The Impact of HS Program on Perinatal Indicators. Maternal and Child Health Journal. 2012.

[16] Slaughter J, Issel LL. Developing a Measure of Prenatal Case Management Dosage. Maternal and Child Health Journal. 2012; 16(5): 1120-1130. PMid:21706295 http://dx.doi.org/10.1007/s10 995-011-0840-7

[17] Kotelchuck, M. Adequacy of Prenatal Care Utilization index: Its U.S. distribution and association with low birth weight. Am J Public Health. 1994; 84: 1486-1489. PMid:8092377 http://dx.doi.org /10.2105/AJPH. 84.9.1486

[18] Field A. Discovering Statistics Using SPSS (and sex and drugs and rock 'n' roll). London: Sage Publications; 2009.

[19] SPSS Inc. PASW Statistics for Windows, Version 18.0. Chicago: SPSS Inc. 2009. 
[20] Wilson Van Voorhis CR, Morgan BL. Understanding Power and Rules of Thumb for Determining Sample Sizes. Tutorials in Quantitative Methods for Psychology. 2007; 3(2): 43-50.

[21] Green SB. How many subjects does it take to do a regression analysis? Multivariate Behavioral Research. 1991; 26: 499-510. http://dx.doi.org/10.1207/s15327906mbr2603_7

[22] Harris RJ. A primer of multivariate statistics (2nd ed.). New York: Academic Press; 1985.

[23] Huber DL, Hall JA, Vaughn T. Dose of case management interventions. Lippincotts Case Management. 2001; 6(3): 119-126. http://dx.doi.org/10.1097/00129234-200105000-00006
[24] Meghea C, Raffo J, Zhu Q, et al. Medicaid home visitation and maternal and infant healthcare utilization. American Journal of Preventive Medicine. 2013; 45(4): 441-447. PMid:24050420 http: //dx.doi.org/10.1016/j.amepre.2013.05.005

[25] Avellar S, Paulsell D. Lessons learned from the Home Visiting Evidence of Effectiveness Review. Office of Planning, Research and Evaluation, Administration for Children and Families, DHHS, Washington DC; 2011.

[26] Darius T, Kathleen P, Constance M, et al. Article: Engagement in Paraprofessional Home Visitation. Families' Reasons for Enrollment and Program Response to Identified Reasons. Women's Health Issues. 2008; 18(2): 118-129. 